\title{
Extra corporeal shockwave lithotripsy resulting in skin burns - a report of two cases
}

\author{
Sandhya R. Rao ${ }^{1}$, Natalia Ballesteros ${ }^{1}$, Kerry L. Short² ${ }^{2}$ Krishna K. Gathani ${ }^{1}$, Murali K. Ankem ${ }^{1}$ \\ ${ }^{1}$ Department of Urology, University of Louisville, Kentucky, USA; ${ }^{2}$ Department of Urology, Robley Rex VA \\ Medical Center, Louisville, Kentucky, USA
}

\section{ABSTRACT}

Severe skin injury after extracorporeal shock wave lithotripsy (ESWL) is rare. We describe two patients who suffered full thickness skin burns following ESWL for renal calculi. One patient was treated conservatively and the other underwent debridement with skin grafting. We speculate that failure of the thermostatic mechanism of the lithotripter, leading to overheating of the water-filled cushion, resulted in this very rare adverse event. Proper preoperative patient counseling regarding the risk of serious burn injuries will help to avoid potential litigation.

\section{ARTICLE INFO}

\section{Key words:}

skin, burn, lithotripsy,

complication

Int Braz J Urol. 2014; 40: 853-7

Submitted for publication:

December 17, 2013

Accepted after revision:

March 16, 2014

\section{INTRODUCTION}

Extracorporeal shock wave lithotripsy (ESWL) is well established as a safe and efficacious mode of treatment of renal and upper ureteric calculi. Despite the low morbidity, a few rare serious adverse events have been reported in the literature. We report an unusual complication in two patients following ESWL which resulted in full thickness skin burns to the flank.

\section{CASE REPORT}

Case 1 - The patient was a 59 year old female with a $5 \mathrm{~mm}$ right lower calyceal calculus and recurrent UTI. She had a history of nephrolithiasis for which she had undergone multiple interventions in the past including ureteroscopy with laser lithotripsy and ESWL. She had multiple co-morbidities including poorly controlled type II diabetes, pulmonary hypertension, hypothyroidism, breast malignancy, osteoarthritis, GERD, obesity, atrial fibrillation. Medications included metformin, insulin, diltiazem, hydralazine, lisinopril, Synthroid ${ }^{\circledR}$, metoprolol and sotalol.

The lithotripter used was the LithoTron ${ }^{\circledR}$ (Healthtronics, Atlanta, GA). The coupling medium used was the Sonotech Clear Image ${ }^{\circledR}$ ultrasound gel, a high viscosity gel containing no bubbles. General anesthesia was induced and the 
patient was positioned appropriately over the lithotripter. With the stone adequately localized under fluoroscopy in the right lower calyx, 3,000 shocks were delivered at a rate of 120 shocks per minute at a power of $24 \mathrm{kV}$. The lithotripter was operated by an experienced lithotripsy technician and the urologist was present in the room throughout the entire procedure.

Immediately after the procedure, following removal of the shock-head, the patient was found to have minor erythematous skin changes with petechiae over the area shocked. She then developed blisters and worsening pain and was admitted overnight for observation. She was discharged home the following day with instructions for daily dressings. She was seen in clinic four days later with bullous lesions and a $10 \mathrm{x}$ $12 \mathrm{~cm}$ superficial burn of the right flank. KUB showed stone clearance and the patient was discharged with instructions for Mepilex ${ }^{\circledR}$ dressing every other day.

The patient returned to the ER five days later with increasing pain. Examination showed significant change in skin appearance with necrosis and eschar formation. The patient was hospitalized and underwent wound debridement for what was now recognized to be a full thickness skin burn. She was discharged home a week later with wound V.A.C therapy and a month later un- derwent successful split thickness skin grafting. Six months later, the flank area was well healed. She continues to experience pain which is treated with gabapentin.

Case 2 - The patient was an asymptomatic 60 years old female with a history of melanoma who underwent surveillance imaging and was incidentally found to have a $1.1 \mathrm{~cm}$ left ureteropelvic junction renal calculus with significant hydronephrosis on CT scan. Other medical history included CVA, goiter, gallstones and psoriasis. Her medications included gabapentin, omeprazole, and lisinopril.

The patient underwent ESWL under general anesthesia using the same machine and coupling gel. As in the first patient, once in appropriate position with stone localized, 3,000 shocks were delivered at 120 shocks per minute and 24 $\mathrm{kV}$. The machine was operated by the same technician as in the first case and once again the urologist was present throughout the procedure.

Upon removal of the lithotripter, a large raised erythematous area was noted on the left flank corresponding to the area of contact with the water-filled cushion. There were a few superficial bullae also visible (Figure-1). She was admitted and treated with daily silver sulfadiazine (Silvadene ${ }^{\circledR}$ ) cream and Adaptic ${ }^{\circledR}$ dressings.

Figure 1 - Large raised erythematous area with superficial bullae seen on post-operative day 2.

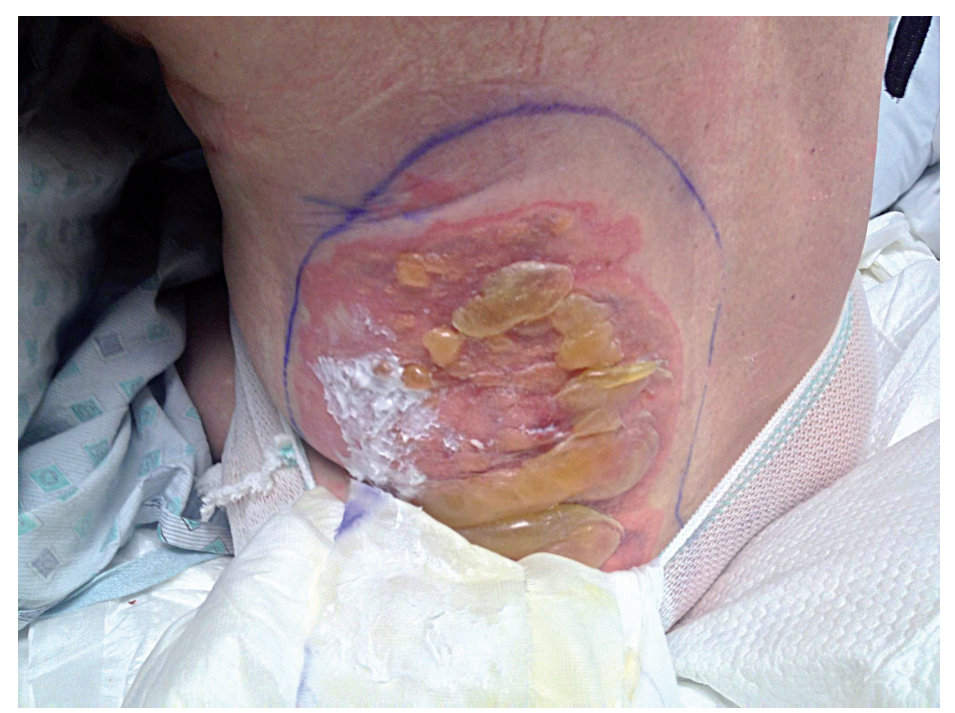


CT scan of the abdomen performed on postoperative day 2 showed an intact stone with no fragmentation and minimal soft tissue stranding along the left flank. There was no evidence of injury to deeper organs. A decision was made to stent the left kidney and perform percutaneous nephrolithotripsy at a later date after complete healing of the flank burn. She was discharged home a week later.

The general surgery team involved in her care determined that approximately fifty percent of the injury was a full thickness burn. The patient refused operative debridement and skin grafting and continued daily wound care with collagenase Santyl ${ }^{\circledR}$ ointment mixed with Polysporin ${ }^{\circledR}$ and Adaptic ${ }^{\circledR}$ dressings. Five months later, she has had almost complete healing of the burn. No intervention for the calculus is planned until the wound is completely healed and stable for a period of time.

\section{DISCUSSION}

Complications following ESWL, immediate or delayed, can be infectious in nature, related to stone fragments, or due to the tissue effects of ESWL. These include bacteruria (7.7-23.5\%) (1), sepsis $(<1-2.7 \%)$ (1), hematuria, renal hematomas (2), and steinstrasse (2-10\%) (3). Rare serious complications include injury to organs such as colon, small bowel, spleen, and liver. The most common skin injuries are petechiae and ecchymosis.

To our knowledge, this is the first report of full thickness skin burns following ESWL for renal calculi. There have been two previously reported cases of partial thickness skin burns following ESWL. One was of a first degree skin burn with erythema, bruising and slight pain which was treated with Thrombocid $^{\circledR}(0.1 \%)$ (4). The second report by Sur et al. (5) was a case of a second degree skin burn following ESWL using a Medispec ${ }^{\circledR}$ EM1000 lithotripter. The patient had two renal calculi and a total of 4,000 shocks were delivered. Conventional ultrasound gel was used instead of the Medispec-recommended coupling medium, Lithoclear ${ }^{\circledR}$ gel. The authors speculate that the skin burns could be attributed to the number of shocks and bubbles associated with a nonapproved ultrasound gel or due to heat generated from the water-filled cushion. There were no mechanical defects in the specific lithotripter used.

In both our patients, a total of 3,000 shocks were delivered at 120 shocks per minute at a power of $24 \mathrm{kV}$. This is the number of shocks routinely delivered at our institution. Sonotech Clear Image Ultrasound gel ${ }^{\circledR}$ is the coupling medium routinely used with this particular machine. This specific lithotripter was evaluated by the parent company, Healthtronics ${ }^{\circledR}$. The usual temperature inside the water-filled cushion is set at 36 degrees Celsius. It was discovered that the thermistor of the water-filled cushion was malfunctioning. Furthermore, it was found that the backup thermostat had faulty wiring. It was usually set to switch off when the water temperature in the shock head rose above 40 degrees Celsius. We presume that the increased water temperature in the cushion secondary to the malfunctioning thermostat was the cause of the skin burns, in the absence of any other explanation. Interestingly, this malfunction was discovered only after the second patient had suffered injury. In the interval between the two cases, the same technician had performed ESWL on seventeen other patients using the same lithotripter without incident. The lithotripter underwent trimesteral servicing even prior to the complication. Following these two incidents, the company has instituted safeguards to prevent future occurrences. The machine continues to be serviced every three months and both the thermistor and the thermostat are tested at each session.

The cause of the injuries in our report remains speculative. ESWL continues to have low morbidity more than 30 years after its initial implementation. Serious complications are rare and occur in less than $1 \%$ of patients. This report highlights an uncommon but serious complication of ESWL. We recommend an increased awareness of the possibility of this complication for medico-legal purposes. Proper preoperative patient counseling regarding the risk of rare but serious burn injuries will help to avoid potential litigation and malpractice claims. 


\section{REFERENCES}

1. Skolarikos A, Alivizatos G, de la Rosette J. Extracorporeal shock wave lithotripsy 25 years later: complications and their prevention. Eur Urol. 2006; 50: 981-90; discussion 990.

2. Dhar NB, Thornton J, Karafa MT, Streem SB. A multivariate analysis of risk factors associated with subcapsular hematoma formation following electromagnetic shock wave lithotripsy. J Urol. 2004; 172: 2271-4.

3. Madbouly K, Sheir KZ, Elsobky E, Eraky I, Kenawy M. Risk factors for the formation of a steinstrasse after extracorporeal shock wave lithotripsy: a statistical model. J Urol. 2002; 167: 1239-42.
4. Romero Pérez P, Martínez Hernández MC. Skin burn caused by shock-wave lithotripsy. Actas Urol Esp. 2004; 28: 632.

5. Rangarajan $S$ MH, Sur RL. Second-degree burn after shock wave lithotripsy: an unusual complication. BJUIwebsite. 2012 25/04/2012. Available at http://www.bjui.org/ ContentFullitem.aspx?id=806\&SectionType $=1 \&$ title $=$ Seco nd-degree-burn-after-shock-wave-lithotripsy:-an-unusualcomplication

\section{Correspondence address:}

Sandhya R. Rao

Department of Urology, University of Louisville 401 e chestnut street, Louisville, Kentucky, 40202, USA E-mail: sandyrrao@yahoo.co.in 


\section{EDITORIAL COMMENT}

Contrasting the reluctance of many journals to publish case reports these days, the vastness of cyberspace has allowed for the development of a new breed of medical journals, specialized in medical cases, allowing the publication and dissemination of notable case reports. While most are still in their infancy, these journals have the potential to act as large case banks.

Many publishers are now revisiting the important value of case reports and new case report specialized journals are surging in an increasing pace, expanding the room for simple reports with educational value, without compromising the classic journals impact factor.

Case reports usually describe an unusual or novel occurrence that though of anecdotal evidence, have a high sensitivity for detecting novelty and therefore remain one of the cornerstones of medical progress; also they provide many new ideas in medicine (1).

Additionally, case reports can have a tremendous impact on our daily practice, bringing a clear learning point, i.e. alerting others to an unexpected treatment response. In fact, the practice of medicine contains countless examples of elegant medical theories that contradict the best available evidence (2).

In the Editorial for the Challenging Clinical Cases Section announcement, I asked myself: "Why and what to write as "Challenging Clinical Cases" in the evidence based era?" (1).
Actually, one of the main roles of case reports is to call attention to a serious unexpected complication of a well-known treatment modality, which was nicely illustrated by surgical complications derived from a problem with the extra corporeal shockwave lithotripsy (SWL) machine thermostat presented by Rao et al. (3).

Though current lithotripters have alarms and sensors that will keep the operator aware of every malfunctioning item, such complications are worth mentioning during preoperative counseling and merit the attention of urologists when applying SWL. Also, recent guidelines or reviews on SWL should be amended to include remarks regarding skin burns as a SWL procedure rare serious complication.

\section{REFERENCES}

1. Reis LO. Editorial for the Challenging Clinical Cases Section. Int Braz J Urol. 2012;38:142-3.

2. Oliveira Reis $L$. The bedrock of daily practice on urology and evidence based medicine. Actas Urol Esp. 2009 Nov;33(10):1054-6.

3. Rao S, Ballesteros N, Short K, Gathani K, Ankem M. Extra corporeal shockwave lithotripsy resulting in skin burns - a report of two cases. Int Braz J Urol 2014.

Leonardo Oliveira Reis, $M D, P h D$

Challenging Clinical Cases Section Editor

Pontifical Catholic University of Campinas, PUC-Campinas

E-mail: reisleo.l@gmail.com 